\title{
Soluzioni cilindriche nella teoria dei plasmi anisotropi.
}

\author{
Grulro MatTer (Pisa) (*)
}

Sunto - Si determinano delle elassi di soluzioni delle equazioni descriventi nell'ambito della teoria di Chew, Goldberger e Low un plasma anisotropo con condizioni al contorno relative ai due casi: (a) dominio cilindrico indefinito, (b) dominio cilindrico finito. Si danno poi le condizioni necessarie e sufficienti per la stabilità delle soluzioni trovate.

\section{1. - Introduzione.}

Lo scopo del presente lavoro è la ricerca di soluzioni delle equazioni descriventi nell'ambito della teoria di CHew, Goldoberger e Low $(C G L)$ un plasma anisotropo con condizioni al contorno relative ai due casi: (a) dominio cilindrico indefinito, nel qual caso si tiene anche conto delle azioni gravitazionali, (b) dominio cilindrico finito.

Al n. 2 si richiama il sistema di equazioni $C G L$ e vari lavori sall'argomento e al n. 3 la forma linearizzata di dette equazioni nell'ipotesi che il plasma imperturbato sia sottoposto ad un forte campo magnetico uniforme. Dopo aver indicato al n. 4 le condizioni al contorno adottate, al n. 5 si determina la forma generale assunta dalle equazioni del n. 3 in un sistema di coordinate cilindriche ortogonali $(r, \varphi, z)$, senza supporre la simmetria assiale delle perturbazioni. Al n. 6 si elaborano le equazioni trovate nel n. 5 . Al n. 7, esaminardo il caso del cilindro indefinito, si determina esplicitamente una classe di soluzioni delle equazioni indefinite, dalla quale si può poi ricavare una classe di soluzioni che soddisfa le condizioni al contorno del n. 4 e assicura la regolarità sull'asse. In detta classe le perturbazioni $v_{r}$ e $v_{c}$ nella velocità $e$ $b_{r}$ e $b_{\varphi}$ nel campo magnetico nel generico piano $(r, \varphi)$ risultano disaccoppiate dalle rimanenti perturbazioni, e cioè da $v_{*}$, sp (perturbazione nella densità) e $\delta U$ (perturbazione nel potenziale gravitazionale) ( $b_{z}$ risulta stazionaria). Le per. turbazioni $v_{r}, v_{\varphi}, b_{r}$ e $b_{\varphi}$, la cui dipendenza funzionale da $r$ risulta espressa tramite opportune funzioni di Bessel e quella da $z, \varphi$ e $t$ è di tipo esponenziale complesso, sono stabili se e solo se si verifica una condizione (cfr. (7.8)), indipendente dal numero d'onda, ohe coincide con la condizione di assenza di instabilità "hose» per le perturbazioni piane. Le perturbazioni $v_{z}$, $\delta \rho$ e $\delta U$, che risultano piane nella presente classe di soluzioni, sono stabili per

(*) Lavoro eseguito nell'ambito della atficità dei Gruppi di Ricerca Matematiea del C.N.R. presso l'Istituto di Matematiche Applicate della Facoltà di Ingegneria della Università di Pisa. 
tutti i valori del numero d'onda maggiori di un valore critico dato dalla (7.16). Per tutti i valori del numero d'onda minori di quello oritico si presenta instabilità del tipo di Jeans.

Al n. 8 si esamina il caso delle oscillazioni torsionali nel cilindro indefinito Al n. 9 infine, considerando il caso del cilindro finito, si determina, col relativo spettro (discreto) di autovalori, una classe esplicita di soluzioni, soddisfacenti alle condizioni al contorno del n. 4.

\section{2. - Le equazioni idromagnetiche per un plasma anisotropo.}

Il sistema di equazioni idromagnetiche con pressione anisotropa, stabilito da Chew, Goldberger e Low [1] per un plasma rarefatto e privo di urti sottoposto a un forte campo magnetico, $\theta$ il seguente:

$$
\begin{gathered}
\rho \frac{d \boldsymbol{v}}{d t}=-\nabla \cdot \boldsymbol{P}+\frac{1}{4 \pi \mu}(\nabla \times \boldsymbol{B}) \times \boldsymbol{B}+\rho \nabla U, \\
\frac{\partial \boldsymbol{B}}{\partial t}=\nabla \times(\boldsymbol{v} \times \boldsymbol{B}), \\
\frac{\partial \rho}{\partial t}=-\nabla \cdot(\rho \boldsymbol{v}), \\
\nabla^{2} U=-4 \pi G \rho,
\end{gathered}
$$

unitamente ai due invarianti adiabatici:

$$
\begin{aligned}
\frac{d}{d t}\left(\frac{p_{i} B^{2}}{\rho^{3}}\right) & =0, \\
\frac{d}{d t}\left(\frac{p}{\rho B}\right) & =0 .
\end{aligned}
$$

In esse $P$ è il tensore delle pressioni avente la forma

$$
\boldsymbol{P}=p_{1} \boldsymbol{I}+\left(p_{n}-p_{1}\right) \boldsymbol{n} \otimes \boldsymbol{n},
$$

dove $I$ è il tensore fondamentale, $\boldsymbol{n}$ il versore del vettore induzione magnetica $\boldsymbol{B}$ e gli scalari $p_{\|}$e $p_{i}$ sono rispettivamente la pressione parallelamente e ortogonalmente a $\boldsymbol{n}(\otimes$ è simbolo di prodotto tensoriale). $U$ è il potenziale gravitazionale e i rimanenti simboli nelle (2.1)-(2.6) hanno il significato abituale.

Per, una analisi delle equazioni sopraseritte, anche per quanto riguarda il campo della loro applicabilita, e per ulteriori sviluppi sul soggetto, si veda per es. Fenraro [2], Lehnert [3] Cap. V n. 1.3 e 2.2, Thompson [4] Sect. 7.5 e 8.7, Kulskud [5] pp. 91-96, Rudakov e Sagdeev [6], Kadomtsev [7], Rosenbluth e Rostoker [8], Rowlands [9], Macmahon [10], Volkov [11], Frieman, Davidson e Langdon [12], Buneman [13]. (Per una bibliografia sui problemi trattati con l'uso delle equazioni CGL si rimanda a quella indicata in [14], aggiungendovi il recente lavoro [15] di RaO, KaLRa e Talwar). 


\section{3. - Le equazioni linearizzate.}

Supponiamo che il plasma non perturbato sia omogeneo, a riposo e sotto. posto a un forte campo magnetico uniforme $\boldsymbol{B}$ la cui direzione assumiamo lungo l'asse $z$ di una terna $T$ di coordinate cilindriche ortogonali $r, \varphi, z$, di corrispondenti versori $\boldsymbol{e}_{r}, \boldsymbol{e}_{\varphi}, \boldsymbol{e}_{z} ;$ quindi

$$
B \equiv(0,0, B) .
$$

Le equazioni delle piecole perturbazioni

$$
\boldsymbol{v}, \delta \boldsymbol{P}, \delta \boldsymbol{B}, \delta \rho \text { e } \delta U
$$

sono (cfr. GLIDDON [16], Trehan [17] p. 43):

$$
\begin{aligned}
\rho \frac{\partial v}{\partial t}=-\nabla \cdot \delta P+\frac{1}{4 \pi \mu}(\nabla \times \delta B) \times B+\rho \nabla \delta U, \\
\frac{\partial}{\partial t} \delta B=\nabla \times(\boldsymbol{v} \times \boldsymbol{B}), \\
\frac{\partial}{\partial t} \delta \rho=-\rho \nabla \cdot v, \\
\nabla^{2} \delta U=-4 \pi G \delta \rho,
\end{aligned}
$$

unitamente alle

$$
\begin{gathered}
\frac{\delta p_{\|}}{p_{\|}}+2 \frac{\delta B}{B}=3 \frac{\delta \rho}{\rho}, \\
\frac{\delta p_{1}}{p_{1}}=\frac{\delta p}{\rho}+\frac{\delta B}{B} .
\end{gathered}
$$

Usando $(3.5)$ e $(3.6)$ si ricava per la perturbazione $\delta \boldsymbol{P}$ nel tensore delle pressioni:

$$
\begin{aligned}
& \delta \boldsymbol{P}=\left(\frac{p_{1}}{\rho} \delta \rho+\frac{p_{1}}{B} \delta B\right) \boldsymbol{I}+\left(\frac{3 p_{\|}-p_{1}}{\rho} \delta \rho-\frac{2 p_{1}+p_{1}}{B} \delta B\right) \boldsymbol{n} \otimes \boldsymbol{n}+ \\
& +\left(p_{s}-p_{1}\right)(n \otimes \delta n+\delta n \otimes n)
\end{aligned}
$$

con $\delta$ nicavabile dalla

$$
\delta \boldsymbol{B}=\delta B \boldsymbol{n}+B \delta \boldsymbol{n} .
$$

Il sistema (3.1)-(3.4), in eui $\delta \boldsymbol{P}$ è specificato da (3.7) $\Theta(3.8)$, ¿ un sistema lineare determinato di otto equazioni scalari in otto funzioni incognite scalari $(\delta p, \delta U$ e sei da $v$ e $\delta B)$. 
Le perturbazioni $\delta p_{\|}^{*}$ e $\delta p_{1}$ sono poi fornite immediatamente dalle (3.5) e (3.6). Indicheremo nel seguito con $\boldsymbol{b}$ la perturbazione $\delta \boldsymbol{B}$ nel campo magnetico e con $b_{r}, b_{\odot}$ e $b_{z}=\delta B$ le sue componenti fisiche relative alla terna $T$ (proiezioni di $\boldsymbol{b}$ secondo $\boldsymbol{e}_{r}, \boldsymbol{e}_{\varphi}$ ed $\boldsymbol{e}_{z}$ rispettivamente).

\section{4. - Condizioni al contorno.}

Considerando il plasma delimitato da una superficie $\Sigma$ di normale $\boldsymbol{N}$, assumiamo quali condizioni al contorno su $\Sigma$ le

$$
\begin{aligned}
& \boldsymbol{v} \cdot N=0, \\
& \boldsymbol{b} \cdot N=0 .
\end{aligned}
$$

Pex quanto concerne l'uso di condizioni al contorno di questo tipo in magnetofluidodinamica (MFD) $e$ in Fisica del plasma cfr. per es. KuLSRUD [5], Bernstein, Frieman, Kruskal e Kulsrod [18], Jeffrey [19] Cap. II.

Nel caso (di possibile interesse astrofisico) in cui $\Sigma$ sia una superficie cilindrica indefinita di raggio $K$ e generatrici parallele all'asse $z$, le (4.1) e (4.2) richiedono che sia:

$$
\begin{aligned}
& v_{r}(R, \varphi, z, t)=0, \\
& b_{r}(R, \varphi, z, t)=0 .
\end{aligned}
$$

Nel caso (di possibile interesse per plasmi di laboratorio) che il plasma sia racchiuso in un eilindro finito di alteza $d$ e raggio $R$ le (4.1) e (4.2), che si presentano per es. se $\Sigma$ è rigida e perfettamente conduttrice, richiedono che, oltre al verificarsi ải $(4.3)$ e $(4.4)$, sia :

$$
\begin{aligned}
& v_{z}(r, \varphi, 0, t)=v_{z}(r, \varphi, d, t)=0, \\
& b_{z}(r, \varphi, 0, t)=b_{z}(r, \varphi, d, t)=0 .
\end{aligned}
$$

Per quanto riguarda le condizioni $(4.3)$ e (4.4) cfr. anche Chandrasekhar [20] n. 81 e per la (4.6) ibidem n. 42 p. 163.

\section{5. - Le equazioni linearizzate in coordinate cilindriche ortogonali.}

In questo numero si stabilisce la forma che le equazioni linearizzate del n. 3 assumono in coordinate cilindriche ortogonali. Per questo scopo determiniamo anzitutto le componenti fisiche relative a $T$ del vettore $\boldsymbol{w}=\nabla \cdot \delta \boldsymbol{P}$. 
Da (3.7) abbiamo intanto:

$$
\nabla \cdot \delta \boldsymbol{P}=\frac{p_{1}}{\rho} \nabla \delta \rho+\frac{p_{1}}{B} \nabla b_{z}+\left(\frac{3 p_{\|}-p_{1}}{\rho} \frac{\partial}{\partial z} \delta \rho-\frac{2 p_{\|}+p_{1}}{B} \frac{\partial b_{z}}{\partial z}\right) \boldsymbol{e}_{z}+\left(p_{\|}-p_{1}\right) \nabla \cdot S,
$$

con

$$
\boldsymbol{S}=\boldsymbol{n} \otimes \delta \boldsymbol{n}+\delta \boldsymbol{n} \otimes \boldsymbol{n} .
$$

Per ottenere le componenti fisiche relative a $T$ del vettore $\nabla \cdot S$ ricordiamo che, con riferimento ad un sistema di coordinate curvilinee ortogonali $x^{h}$ di coefficienti metrici $h_{k}$, le componenti fisiche $\alpha_{i}$ di un generico vettore a sono date da (cfr. Finzi e Pastoni [21] Cap. II \& 8 n. 5, Sterrin [22] p. 143;:

$$
\alpha_{i}=h_{i} a^{i}=\frac{1}{h_{i}} a_{i} \quad(i \text { non sommato }),
$$

dove $a^{i}$ e $a_{i}$ sono rispettivamente le componenti controvarianti e covarianti di $a$, e inoltre, che sussiste la (SERRIN [22] p. 142)

$$
(\nabla \cdot S)_{i}=\frac{1}{\sqrt{g}} \frac{\partial}{\partial x^{k}}\left(\sqrt{g} S_{i}^{k}\right)-S_{k}^{k} \frac{\partial \log _{i}^{i} h_{k}}{\partial x^{i}} \quad \text { (somma su } k \text { ) }
$$

con

$$
\sqrt{g}=h_{1} h_{2} h_{3}
$$

Nel nostro caso è

$$
h_{\mathrm{I}}=h_{3}=1, h_{2}=r, \sqrt{g^{-}}=r .
$$

Facendo uso di (5.2), (3.8) e (5.3) otteniamo da (5.4) per le componenti fisiche relative a $\mathrm{T}$ di $\nabla \cdot S$ rispettivamente: $\left({ }^{1}\right)$

$$
\frac{1}{B} \frac{\partial b_{r}}{\partial z}, \frac{1}{B} \frac{\partial b_{\varphi}}{\partial z}, \frac{1}{B} \frac{1}{r}\left[\frac{\partial\left(r b_{r}\right)}{\partial r}+\frac{\partial b_{\varphi}}{\partial \varphi}\right]
$$

(1) Alle (5,5) si perviene anche da (5,2) con l'uso della (3,8) tenendo presente che, se c e $d$ sono due generici vettori, sussiste l'identità vettoriale:

$$
\nabla \cdot(c \otimes d)=(c \cdot \nabla) d+(\nabla \cdot c) d
$$

(cfi. Ferraro e Plumpton [23] (8.44)), o che le componenti fisiche relative a $T$ del vettore $(c \cdot \nabla) d$ sono rispettivamente

$$
(\mathbf{c} \cdot \nabla) d_{r}-c_{\varphi} d_{\varphi} / r,(\mathbf{c} \cdot \nabla) d_{\varphi}+c_{\varphi} d_{y} / r,(\mathbf{c} \cdot \nabla) d_{z}
$$

(cfr. Chandrasekhar [20] p. 558, Batchelor [24] p. 602). (Il simbolismo, con le relative definizioni adottato qui e nel resto del lavoro è quello di uso corrente nella letteratura anglo-sassone). 
Da (5.1), tenendo conto delle (5.5), otteniamo allora le cercate componenti fisiche relative a $T$ di $w=\nabla \cdot \delta P$ :

$$
\begin{gathered}
w_{r}=\frac{p_{1}-p_{1}}{B} \frac{\partial b_{r}}{\partial z}+\frac{p_{1}}{\rho} \frac{\partial}{\partial r} \delta \rho+\frac{p_{1}}{B} \frac{\partial b_{z}}{\partial r}, \\
w_{\varphi}=\frac{p_{1}-p_{1}}{B} \frac{\partial b_{\varphi}}{\partial z}+\frac{1}{r} \frac{p_{1}}{\rho} \frac{\partial}{\partial \rho} \delta \rho+\frac{1}{r} \frac{p_{1}}{B} \frac{\partial b_{z}}{\partial \varphi}, \\
w_{z}=\frac{p-p_{1}}{B} \frac{1}{r}\left[\frac{\partial\left(r b_{r}\right)}{\partial r}+\frac{\partial b_{\varphi}}{\partial \varphi}\right]+\frac{3 p_{\|}}{\rho} \frac{\partial}{\partial z} \delta \rho-\frac{2 p_{1}}{B} \frac{\partial b_{z}}{\partial z} .
\end{gathered}
$$

Denotando con $v_{r}, v_{\varphi}$ e $v_{z}$ le componenti fisiche di $v$ relative a $T$ e facendo uso delle $(5.6),(5.7)$ e $(5.8)$, la (3.1) proiettata su $T$ dà origine alle tre equazioni scalari :

$$
\begin{aligned}
& \text { (5.9) } \rho \frac{\partial v_{r}}{\partial t}+\left(\frac{p_{1}}{B}+\frac{B}{4 \pi \mu}\right) \frac{\partial b_{z}}{\partial r}+\left(\frac{p_{\|}-p_{1}}{B}-\frac{B}{4 \pi \mu}\right) \frac{\partial b_{r}}{\partial z}+\frac{p_{1}}{\rho} \frac{\partial}{\partial r} \delta \rho-\rho \frac{\partial}{\partial r} \delta U=0, \\
& \text { (5.10) } \rho \frac{\partial v_{\varphi}}{\partial t}+\left(\frac{p_{\|}-p_{1}}{B}-\frac{B}{4 \pi \mu}\right) \frac{\partial b_{\varphi}}{\partial z}+\frac{p_{1}}{\rho} \frac{1}{r} \frac{\partial}{\partial \varphi} \delta \rho+\frac{1}{r}\left(\frac{p_{1}}{B}+\frac{B}{4 \pi \mu}\right) \frac{\partial b_{z}}{\partial \rho}-\frac{\rho}{r} \frac{\partial}{\partial \varphi} \delta U=0, \\
& \text { (5.11) } \quad \rho \frac{\partial v_{z}}{\partial t}+\frac{p_{\|}-p_{1}}{B} \frac{1}{r}\left[\frac{\partial\left(r b_{r}\right)}{\partial r}+\frac{\partial b_{\rho}}{\partial \varphi}\right]-\frac{2 p_{1}}{B} \frac{\partial b_{z}}{\partial z}+\frac{3 p_{\|}}{\rho} \frac{\partial}{\partial z} \delta \rho-\rho \frac{\partial}{\partial z} \delta U=0,
\end{aligned}
$$

Dalla (3.2) abbiamo:

$$
\begin{gathered}
\frac{\partial b_{r}}{\partial t}-B \frac{\partial v_{r}}{\partial z}=0, \\
\frac{\partial b_{\varphi}}{\partial t}-B \frac{\partial v_{\varphi}}{\partial z}=0, \\
\frac{\partial b_{z}}{\partial t}+\frac{B}{r}\left[\frac{\partial v_{\varphi}}{\partial \varphi}+\frac{\partial\left(r v_{r}\right)}{\partial r}\right]=0,
\end{gathered}
$$

mentre le $(3.3)$ e (3.4) forniscono rispettivamente:

$$
\begin{gathered}
\frac{\partial}{\partial t} \delta \rho+\rho\left[\frac{\partial v_{z}}{\partial z}+\frac{1}{r} \frac{\partial\left(r v_{v}\right)}{\partial r}+\frac{1}{r} \frac{\partial v_{\varphi}}{\partial \varphi}\right]=0, \\
\frac{1}{r} \frac{\partial}{\partial r}\left(r \frac{\partial}{\partial r} \delta U\right)+\frac{1}{r^{2}} \frac{\partial^{2}}{\partial \varphi^{2}} \delta U+\frac{\partial^{2}}{\partial z^{2}} \delta U+4 \pi G \delta \rho=0 .
\end{gathered}
$$


Le (5.9)-(5.16) sono, in coordinate cilindriche ortogonali, le cercate otto equa.

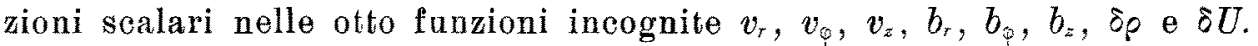

\section{6. - Elaborazione delle equazioni trovate.}

Dalle $(5.9),(5.10),(5.11)$ e $(5.16)$ si possono eliminare $b_{r}, b_{\varphi}, b_{z}$ e $\delta \rho$ facendo uso delle (5.12), (5.13), (5.14) e (5.15).

Si ottengono così le:

$$
\begin{gathered}
\frac{\partial^{2} v_{r}}{\partial t^{2}}-\frac{h \partial^{2} v_{r}}{\partial z^{2}}-\frac{2\left(p_{m}+p_{1}\right)}{\rho} \frac{\partial}{\partial r} \nabla \cdot v_{1}-\frac{p_{1}}{\rho} \frac{\partial^{2} v_{z}}{\partial r \partial z}-\frac{\partial^{2}}{\partial l i r} \delta U=0, \\
\frac{\partial^{2} v_{\varphi}}{\partial t^{2}}-\frac{h}{\rho} \frac{\partial^{2} v_{\varphi}}{\partial z^{2}}-\frac{2}{r} \frac{p_{1}+p_{m}}{\rho} \frac{\partial}{\partial \varphi} \nabla \cdot v_{1}-\frac{1}{r} \frac{p_{1}}{\rho} \frac{\partial^{2} v_{z}}{\partial \varphi \partial z}-\frac{1}{r} \frac{\partial^{2}}{\partial t \partial \varphi} \delta U=0, \\
\frac{\partial^{2} v_{z}}{\partial t^{2}}-\frac{3 p_{11}}{\rho} \frac{\partial^{2} v_{z}}{\partial z^{2}}-\frac{p_{1}}{\rho} \frac{\partial}{\partial z} \nabla \cdot v_{1}-\frac{\partial^{2}}{\partial t \partial z} \delta U=0, \\
\frac{\partial}{\partial t}\left[\frac{1}{r} \frac{\partial}{\partial r}\left(r \frac{\partial}{\partial r} \delta U\right)+\frac{1}{r^{2}} \frac{\partial^{2}}{\partial \varphi^{2}} \delta U+\frac{\partial^{2}}{\partial z^{2}} \delta U\right]-4 \pi G \rho \nabla \cdot v=0,
\end{gathered}
$$

dove si è posto:

$$
h=2 p_{m}+p_{1}-p_{n},
$$

con

$$
p_{m}=B^{2} / 8 \pi \mu,
$$

pressione magnetica, e si è indicato con

$$
v_{1}=v_{r} \boldsymbol{e}_{r}+v_{\varphi} \boldsymbol{e}_{\varphi}
$$

il componente di $\boldsymbol{v}$ normale a $\boldsymbol{B}$.

Una volta determinata per $v$ e $\delta U$ una soluzione del sistema $(6.1)-(6.4)$, da $(5.12)-(5.15)$ si ricavano, in corrispondenza, $\boldsymbol{b}$ e $\delta \rho$ con delle quadrature.

Nei n. 7 e 9 si determinano alcune classi di soluzioni soddisfacenti alle condizioni al contorno indicate al n. 4.

\section{7. - Una classe di soluzioni per il cilindro indefinito.}

Una classe di soluzioni del sistema $(6.1)-(64)$ si può determinare nel modo seguente.

Richiediamo che $v_{r}$ e $v_{\varphi}$, pur essendo funzioni di tutte e tre le variabili spaziali e del tempo, diano origine, nel generico piano ortogonale a $\boldsymbol{B}$, a un 
vettore solenoidale $\left(\nabla \cdot v_{1}=0\right)$ e che $v_{z}$ e $\delta U$ siano fanzioni solo di $z$, oltre che del tempo. In tal caso le perturbazioni $v_{r} \otimes v_{\varphi}$ sono disaccoppiate dalle altre e devono essere soluzioni delle

$$
\frac{\partial^{2} v_{r}}{\partial t^{2}}=\frac{h}{\rho} \frac{\partial^{2} v_{r}}{\partial z^{2}}
$$

$$
\frac{\partial^{2} v_{\varphi}}{\partial t^{2}}=\frac{h}{\rho} \frac{\partial^{2} v_{\varphi}}{\partial z^{2}}
$$

soddisfacenti al vincolo

$$
\nabla \cdot \boldsymbol{v}_{1} \equiv \frac{1}{r}\left[\frac{\partial\left(r v_{r}\right)}{\partial r}+\frac{\partial v_{0}}{\partial \varphi}\right]=0
$$

le perturbazioni $v_{z}$ e $\delta U$ soddisfano alle

$$
\begin{gathered}
\frac{\partial^{2} v_{z}}{\partial t^{2}}=\frac{3 p_{i}}{\rho} \frac{\partial^{2} v_{z}}{\partial z^{2}}+\frac{\partial^{2}}{\partial t \partial z} \delta U \\
\frac{\partial^{3}}{\partial t \partial z^{2}} \delta U=4 \pi G_{\rho} \frac{\partial v_{z}}{\partial z}
\end{gathered}
$$

La (7.3) resta identicamente soddisfatta dalla

$$
\left\{\begin{array}{l}
v_{r}=\bar{v}_{r} \frac{m}{r} \psi(r) e^{i(\omega t-k x-m \varphi)} \\
v_{\varphi}=-i \bar{v}_{r} \frac{d \psi(r)}{d r} e^{i(\omega t-k=-m \varphi)}
\end{array}\right.
$$

di cui è chiaro il significato fisico $\left[\bar{v}_{r}\right.$ è una costante, $\psi$ una arbitraria funzione di $r$, $\omega$ la pulsazione (che potrà risultare complessa), $m$ un intero (positivo o negativo) e $k$ è il numero d'onda relativo alla direzione $z$ ( $k$ lo consideriamo prefissato reale)].

Le $(7.1)$ e (7.2) ammettono la soluzione (7.6) col verificarsi della relazione di dispersione, indipendente da $m$,

$$
\omega^{2}=\frac{h}{\rho} k^{2} .
$$

Se è

$$
h>0
$$


le perturbazioni $v_{r}$ e $v_{\varphi}$ si propagano con la velocità di fase reale $u=\omega / k$ data dalla

$$
u^{2}=A^{2}+\frac{p_{1}-p_{\|}}{\rho}
$$

dove $A^{2}=B^{2} / 4 \pi \mu \rho$ è il quadrato della velocità di Alfvén. La (7.9) caratterizza onde di Alfvén modificate a causa dell'anisotropia nel tensore delle pressioni.

Se ̀̀

$$
h<0
$$

da (7.7) si hanno per $u$ due valori immaginari coniugati e ciò è collegato con un noto fenomeno di instabilità nel plasma in esame. Infatti una condizione identica alla (7.10) caratterizza il verificarsi della cosidetta instabilita "hose» delle piccole perturbazioni piane nel plasma indefinito (ofr. per es. LEHNERT [3] pp. 146-48). ( ${ }^{2}$ )

Per quanto riguarda la determinazione di $\boldsymbol{b}$, possiamo intanto prescindere dalla perturbazione $b_{z}$ in quanto la (5.14) indica che, per (7.3), essa è stazionaria. Per le perturbazioni $b_{r}$ e $b_{\varphi}$ le (5.12) e (5.13) forniscono in corrispondenza alle (7.6) le:

$$
\left\{\begin{array}{l}
b_{r}=-\frac{B k}{\omega} v_{r}+f_{1}(r, \varphi, z) \\
b_{\varphi}=-\frac{B k}{\omega} v_{\varphi}+f_{2}(r, \varphi, z) .
\end{array}\right.
$$

In queste $f_{1}$ e $f_{2}$ sono funzioni arbitrarie di $r, \varphi$ e $z$ dalle quali si può pre. scindere data la loro stazionarietà con il che resta identicamente soddisfatta la condizione che richiede la solenoidalità di $\boldsymbol{b}$.

Con le soluzioni (7.6), (7.11) possiamo soddisfare alle condizionî al contorno $(4.3)$ e (4.4) relative al cilindro indefinito specializzando la funzione arbitraria $\psi(r)$ in

$$
\psi(r)=J_{m}\left(\alpha_{m s} \frac{r}{R}\right)
$$

(2) Per quanto riguarda questo tipo di instabilità si vella anche per es. Parker [25], Chandraserhar, Kaummann, Watson [26] p. 450, Lus't [27], Thompson [4] p. 216, Gimdonon [16], Kato, Tajiri e Taniuti [28], Abraham-Shirauner [29], Vhdenov e Sagdeev [30], e il precedente lavoro [31]. 
dove $J_{m}$ è la funzione di Bessel di prima specie di ordine $m$ e $\alpha_{m s}$ il suo s-mo zero $(s=1,2,3, \ldots)$. In corrispondenza a $(7.12)$ la $(7.6)_{2}$ asume la forma:

$$
v_{\phi}=i \bar{v}_{r}\left[\frac{m}{r} J_{m}\left(\alpha_{m s} \frac{r}{R}\right)-\frac{\alpha_{m s}}{R} J_{m-1}\left(\alpha_{m s} \frac{r}{R}\right)\right] e^{i(\omega t-k z-m \varphi)}
$$

La scelta (7.12) assicura inoltre la regolarità sull'asse delle soluzioni (7.6), (7.11). Venendo ora alle (7.4) e (7.5) esse ammettono la soluzione:

$$
\left\{\begin{array}{l}
v_{z}=\bar{v}_{z} e^{i\left(\omega_{*} t-k_{*} z\right)} \\
\delta U=\overline{\delta U} e^{i\left(\omega_{*} t-k_{*} z\right)}
\end{array}\right.
$$

di significato fisico evidente, fornendo in corrispondenza la relazione di dispersione

$$
\omega_{*}^{2}=\frac{3 p_{\| 1}}{\rho} k_{*}^{2}-4 \pi G \rho
$$

La (7.15) mette in evidenza che per tutti i valori del numero d'onda $k_{*}$ inferiori al valore critico

$$
k_{*}^{c}=\left(\frac{4 \pi G \rho}{3 p_{11} / \rho}\right)^{1 / 2}
$$

sorge nel plasma in esame, in corrispondenza alle perturbazioni piane (7.14), instabilita gravitazionale del tipo di Jeans. Per tutti $i$ valori del numero d'onda invece che verificano la condizione

$$
k_{*}>k_{*}^{e}
$$

c'è stabilità gravitazionale.

Il valore (7.16) del numero d'onda oritico coincide con quello determinato da GLIDDON [16] nello studio della instabilità gravitazionale nel plasma inde. finitn, relativamente a perturbazioni piane propagantesi lungo $\boldsymbol{B}$. In corrispon. denza alla $(7.14)_{x}$, la $(5.15)$, per (7.3), fornisce per $\delta \rho$ l'espressione

$$
\delta \rho=\frac{\rho k_{*}}{\omega_{*}} v_{z}
$$

a meno di un campo stazionario

Concludendo: per un plasma anisotropo priro di urti descritto dalle equazioni linearizzate di CHEw, GoLDBERGER e Low, autograxitante in un dominio cilindrico indefinito con generatrici parallele al campo magnetico $\boldsymbol{B}$, 
sussiste, soddisfacente alle condizioni al contorno (4.3) e (4.4), la seguente classe di soluzioni :

$$
\begin{aligned}
& v_{r}=\bar{v}_{r} \frac{m}{r} J_{m}\left(\alpha_{m s} \frac{r}{R}\right) e^{i(\omega t-k s-m \varphi)} \\
& v_{\varphi}=i \vec{v}_{r}\left[\frac{m}{r} J_{m}\left(\alpha_{m s} \frac{r}{R}\right)-\frac{\alpha_{m s}}{R} J_{m-1}\left(\alpha_{m s s} \frac{r}{R}\right)\right] e^{i(\omega t-k z-m \varphi)} \\
& b_{r}=-\frac{B k}{\omega} v_{r} \\
& b_{\varphi}=-\frac{B k}{\omega} v_{\varphi}
\end{aligned}
$$

unitamente alle:

$$
\begin{aligned}
& v_{z}=\bar{v}_{z} e^{i\left(\omega_{*} t-k_{*} z\right)} \\
& \delta U=\overline{\delta U} e^{i\left(\omega_{*} t-k_{*} z\right)} \\
& \delta \rho=\frac{\rho k_{*}}{\omega_{*}} v_{z},
\end{aligned}
$$

con il presentarsi delle due relazioni di dispersione (7.7) e (7.15).

Le (7.19) foruiscono le perturbazioni nella velocità e nel campo magnetico nel generico piano $(r, \wp)$ normale a $B$; esse, nella presente classe di soluzioni, risultano disaccoppiate dalle rimanenti perturbazioni $v_{z}$, $\delta \rho$ e $\delta U$, fornite dalle (7.20). $\left({ }^{3}\right)$ Queste soluzioni, ohe assicurano la regolarità sull'asse, sono stabili se e solo se sono verificate le due condizioni (7.8) e (7.17), la prima delle quali, indipendente dal numero d'onda, è legata all'anisotropia nel tensore delle pressioni, e la seconda all'azione gravitazionale. $\left({ }^{4}\right)$

\section{8. - Oscillazioni torsionali nel cilindro indefinito.}

Ferme restando le soluzioni $(7.20)$ per le perturbazioni $v_{z}$, $\delta p$ e $\delta U$, con la relativa relazione di dispersione (7.15), consideriamo il caso in cui sia identicamente

$$
v_{r}=b_{r}=0
$$

(3) Per quanto riguarda la perturbazione $b_{z}$ si è preseisso da essa data la sua stazionarieta nella presente classe di soluzioni.

(4) Kicordiumo che nella zona di totale iperbolicità del sistema di equazioni $C G L$ è assicurato il verificarsi della condizione (7.8) (cfr. [28], [29], [31]). 
e che le rimanenti perturbazioni $v_{\varphi}$ e $b_{\varphi}$ presentino simmetria assiale (5), $\left({ }^{6}\right)$; è questo un caso collegato con lo studio delle oscillazioni torsionali. (Per quanto riguarda tale tipo di oscillazioni in MFD quando, oltre alle (8.1), ̇̀ anche $v_{z}=0$, efr. per es. Ferraro e Plumpton [23] pp. 86 e segg., dove è esaminato il caso di un fluido incomprimibile non viscoso, ma di conducibilità elettrica finita, in assenza di forze di massa di natura non elettromagnetica).

Nel caso presente $x_{\varphi}$ e $b_{\varphi}$ (efr. (5.10) e (5.13)) sono disaccoppiate dalle rimanenti perturbazioni e soddisfano entrambe alla stessa equazione:

$$
\frac{\partial^{2} v_{\varphi}}{\partial t^{2}}=\frac{h}{\rho} \frac{\partial^{2} v_{\varphi}}{\partial z^{2}}, \frac{\partial^{2} b_{\varphi}}{\partial t^{2}}=\frac{h}{\rho} \frac{\partial^{2} b_{\varphi}}{\partial z^{2}}
$$

Le (8.2) forniscono le soluzioni

$$
v_{\varphi}=f(r) e^{i\left(\omega^{\prime} t-k^{\prime} z\right)}, b_{\varphi}=g(r) e^{i\left(\omega^{\prime} t-k^{\prime} z\right)},
$$

dove $f$ e $g$ sono arbitrarie funzioni di $r$ e $\omega^{\prime}$ (pnlsazione) e $k^{\prime}$ (numero d'onda) sono legati da una relazione di dispersione amaloga alla (7.7).

Se, seguendo gli Autori di [23], assumiamo quale condiziono al contorno per $\boldsymbol{b}$ la:

$$
b_{\varphi} \equiv b=0 \text { per } r=R
$$

questa, giovandoci della arbitrarietà della $g(r)$, può soddisfarsi per es. prendendo la soluzione, regolare sull'asse,

$$
b=\bar{b} J_{\nu}\left(\alpha_{\nu q} \frac{r}{R}\right) e^{i\left(\omega^{\prime} t-k^{\prime}=\right)},
$$

dove $\bar{b}$ ò una costante e, al solito, $J_{\nu}$ è la funzione di Bessel di prima specie di ordine $\vee$ e $\alpha_{v q}$ il suo $q$-mo zero $(q=1,2,3, \ldots)$. In corrispondenza alla (8.4), la (5.10) fornisce, a meno del solito campo stazionario, la

$$
v_{\varphi}=-\frac{k^{\prime}}{\omega^{\prime} \rho}\left(\frac{B}{4 \pi \mu}+\frac{p_{1}-p_{\|}}{B}\right) b
$$

(5) Per la perturbazione $b_{z}$ abbiamo da (5.14) $\frac{\hat{\partial} b_{z}}{\hat{c} t}=0$ e quindi anche qui possiamo pre. scindere da essa. Ia condizione di solenoidalita di $b$ resta poi identicamente soddisfatia.

(6) Una classe di soluzioni del sistema (5.9)-(5.16) soddisfacenti alle eundizioni al contorno (4.3) e (4.4) è stata determinata in un precedente luroro [32] sotto la sola ipotesi di simmetria assiale per le perturbazioni. 


\section{9. - Caso del cilindro finito.}

In questo caso, di possibile interesse per plasmi di laboratorio, trascuriamo, come è consueto, le forze gravitazionali (e ogni altra forza di massa di origine non elettromagnetica) rispetto a quelle elettromagnetiche. Il sistema di equa. zioni differenziali indefinite è ora costituito dalle $(6.1),(6.2)$ e (6.3) (senza i termini gravitazionali) nelle incognite $v_{r}, v_{\varphi}$ e $v_{z}$ (nota $v$ le (5.12)-(5.15) fornicono anche qui b e óp per quadrature); le condizioni al contorno sono espresse dalle (4.3), (4.4), (4.5) e (4.6).

Una classe di soluzioni per il caso in questione si ottiene nel modo seguente.

Per $v_{r}, v_{\varphi}, b_{r}$ e $b_{\varphi}$ prendiamo le soluzioni (7.19), che soddisfano alle (6.1), (6.2), (5.12) e (5.13) e alle condizioni al contorno (4.3) e (4.4); corrispondentemente da (5.14) e dalla condizione di solenoidalità di $\boldsymbol{b}$ abbiamo

$$
\frac{\partial b_{z}}{\partial t}=\frac{\partial b_{z}}{\partial z}=0
$$

e queste, con le condizioni al contorno (4.6), sono soddisfatte identicamente da $b_{z} \equiv 0$. Per quanto riguarda $v_{z}$ una soluzione della (6.3) soddisfacente alle condizioni al contorno (4.5) è data dalla

$$
v_{z}=\bar{v}_{z} \sin \frac{n \pi}{d} z \sin \left(\omega_{*} t-\alpha\right) \quad(n=1,2,3, \ldots),
$$

di significato fisico evidente, in corrispondenza alla quale abbiamo lo spettro discreto di autovalori reali

$$
\omega_{* n}= \pm \frac{n \pi}{d}\left(\frac{3 p_{\mid i}}{\rho}\right)^{1 / 2}
$$

Per la perturbazione op infine la (5.15), fornisce a meno del solito campo stazionario, la

$$
\delta \rho=\frac{\rho \bar{v}_{x}}{\omega_{*}} \frac{n \pi}{d} \cos \frac{n \pi}{d} z \cos \left(\omega_{*} t-\alpha\right)
$$

Osservazione. - Se le condizioni al contorno richiedessero, oltre alle (4.3), (4.4), (4.5) e (4.6), l'annullamento di tutto il vettore $v$ sulle basi del ci. lindro, ciò̀ il verificarsi delle ulteriori condizioni

$$
v_{r}(r, \varphi, 0, t)=v_{\varphi}(r, \varphi, 0, t)=v_{r}(r, \varphi, d, t)=v_{\varphi}(r, \varphi, d, t)=0,
$$

una classe di soluzioni, regolari sull'asse, si determinerebbe facilmente pren- 
dendo per $v_{z}$ e $\delta \rho$ le $(9.1)$ e $(9.3)$ e per $v_{r}$ e $v_{\varphi}$, in luogo delle $(7.19)_{1,2}$, le:

$(*)$

$$
v_{r}=\bar{v}_{r} \frac{m}{r} J_{m s}\left(\alpha_{m s} \frac{r}{R}\right) \sin \left(\frac{k \pi}{d} z\right) e^{i(10 t-m \varphi)}
$$

r

$$
\begin{gathered}
v_{\varphi}=i \bar{v}_{r}\left[\frac{m}{r} J_{m}\left(\alpha_{m i s} \frac{r}{R}\right)-\frac{\alpha_{m s}}{R} J_{m-1}\left(\alpha_{m s} \frac{r}{R}\right)\right] \sin \left(\frac{k \pi}{d} \approx\right) e^{i(\omega t-m \varphi)} \\
(k=1,2,3, \ldots)
\end{gathered}
$$

in corrispondenza alle quali abbiamo (cfr. (7.1) e (7.2)) lo spettro discreto di autovalori (reali nell'ipotesi di assenza di instabilità «hose»)

$$
\omega_{k}= \pm \frac{k \pi}{d}\left(\frac{h}{\rho}\right)^{1 / 2}
$$

Per quanto riguarda $b_{r}$ e $b_{\phi}$ infine abbiamo da (5.12) e (5.13), in corrispondenza alle (*), :

$$
\left\{\begin{array}{l}
b_{r}=\frac{B \overline{v_{r}}}{i \omega} \frac{k \pi}{d} \frac{m}{r} J_{n}\left(\alpha_{m s} \frac{r}{R}\right) e^{i(\omega t-m \varphi)} \cos \left(\frac{k \pi}{d} z\right) \\
b_{\varphi}=\frac{\overrightarrow{B v_{r}^{i}}}{\omega} \frac{k \pi}{d}\left[\frac{m}{r} J_{m}\left(\alpha_{m s} \frac{r}{R}\right)-\frac{\alpha_{m s}}{R} J_{m-1}\left(\alpha_{m s} \frac{r}{R}\right) \mid e^{i(\omega t-m \varphi)} \cos \left(\frac{k \pi}{d} z\right)\right.
\end{array}\right.
$$

a meno del solito campo stazionario.

\section{BIBLIOGRAFIA}

[1] G. F. Chew, M. L. GoLdBerGer, F. E. Low, The Boltzmann equation and one fluid hydromagnetic equations in the a'sence of particle collisions, Proc. Roy. Soc., A 236, (19506), pp. 112-118.

[2] V. C. A. Ferraro, Lezioni sulla dinamica dei gas ionizzati, Istituto Matematico Università di Firenze, (1967).

[3] B. LeHNert, Dynamics of charged particles, North-Holland Publ. Co., Amsterdam, (1964).

[4] W. B. Thompsos, An introduction to plasma Physics, Pergamon Press, (1962).

[5] R. KULsRud, General stability theory in plasma Physics, Rend. Scuola Int di Fisica E. Fermi, XXV, Academic Press, (1964), pp. 54-96.

[6] L. I. Rudakov, R. Z. SAGDEEv, A quasi-hydrodynamic description of a rarefied plasma in a magnetic field, Plasma Physics and problems of controlled thermonuclear reactions, III, Pergamon Press, (1959), pp. 321-3331.

[7] B. B. Kadomtsev, Plasma dynamics in a strong magnetic field, ibidem IV, (1960), pp. 438-449. 
[8] M. N. Rosenbluti, N. Rostorer, Theoretical structure of plasma equations, Phy. Elu. ids, 2, (1959), pp. 23-30.

[9] G. Row Lands, Some aspects of theoretical plasma Physics in the United Kingdom, Propagation and instabilities in plasmas, Stanford University Press, (1963), pp. 52-69.

[10] A. МасманоN, Finite gyro-radius corrections to the hydramagnetic equations for a Vlasov plasma, Phy. Fluids, 8, (1955), pp. 1840-5.

[11] T. F. Vockov, Hydrodynamic description of a collisionless plasma, Reviews of plasma Physics, Ed. Leontovich, 4, pp. 1-21, Consultants Bureau, New York, (1966).

[12] E. Frieman, R. Davidson, B. Langdon, Higher order corrections to the Chew-Goldberger -Low theory, Phy. Fluids, 9, (1966), pp. 1475-82.

[13] O. Bungman, Gas law and conductivity of a collision-free plasma, Phy. Fluids, 4, (1661); pp. $669-680$.

[14] G. MATTEI, Wave propagation and instabilities in a rotating anisotropic plasma in corso di pubblicazione su Meccanica.

[15] S. S. RAO, G. L. KALRA, S. P. TALWAR, Slipping stream instability in anisotropic plasma with magnetic shear, Jour. Plasma Phy., 2, (1968), pp. 181-188.

[16] J. E. C. Gruddon, Gravitational instability of anisotropic plasma, Astropby. Jour., 145, (1966), pp. 583-588.

[17] S. K. Trehan, Macroscopic thuory of plasma waves, Plasma Physics, AIEA, Vienna (1965).

[18] I. B. Bernstein, E A. Frigman, M. D. Kruskal, R. M. Kursrud, An energy principle for hydromagnetic stability problems, Proe. Roy. Soc., A 244, (1958), pp. 17-40.

[19] A. JefFrey, Magnetohydrodynamics, Oliver and Boyd, London, (1966).

[20] S. Chanorasekhar, Hydrodynamie and hydromagnetic stability, Oxford, (1961).

[21] B. Frnzi, M. Pastori, Calcolo tensoriale e applicazioni, Zanichelli. Bologna, (1961).

[22] J. Serrin, Mathematical principles of classical fluid Mechanics, Hand. Phy. VIII/1, (1959).

[23] V. C. A. Ferraro, C. Plumptos, An introduction to magnetotiuid mechanics, II Ed. Oxford, (1966).

[2t] G. K. Batrohelor, An introduction to fluid dynamies, Cambrilge Univ. Press, (1967).

[25] E. N. PARKER, Dynamical instability in an anisotropic ionized gas of low density, Phys. Rev., 109, (1958), pp. 1874-76.

[26] S. Chandrasekgar, A. N. Kaufmann, K. M. Watson, the stability of the pinch, Proc. Roy. Soc., A 245, (1958), pp. 435-455.

[27] R. Lust, Int. Summer course in plasma Physics, Ed. C. F. Wandel, Danish Atomic Energy Commission, (1960), p. 201.

[28] Y. Като, M. TaJir, T. TANIUTI, Propagation of hydromagnetic waves in collisionless plasma I, Jour Phy. Soc. Japan, 21, (1966), pp, 765-777.

[29] B. Abramam-Shrauner, Propagation of hydromagnetic waves through an anisotropic plasma, Jour. Plasma Phy., 1, (1967), pp. 361-378.

[30] A. A. Vedevov, R. Z. SAgDeEV, Some properties of a plasma with an anisotropic ion velocity distribution in a magnetic field, Plasma Physics and problems of controlled ther. monuclear reactions III, Peigamon Press, Oxford, (1959), pp. 332-339.

[31] G. MATTEI, Varietà caratteristiche e propagazione ondosa in un plasma magnetizzato privo di urti, Annali Sc. Normale Pisa, XXI, (1967), pp, 745-763.

[32] G. Matrer, On the propagation of cylindrical waves in a magnetized self-gravitating collisionless plasma, Jour. Plasma Phy., 2, (1968), pp. 9-15. 